\title{
Modelling deformation and fracture in confectionery wafers
}

\author{
Idris K. Mohammed, Maria N. Charalambides, J. Gordon Williams, John Rasburn
}

Mechanical Engineering Department, Imperial College London, South Kensington, London, SW7 2AZ, Nestec York Ltd., Nestlé Product Technology Centre, Haxby Road, PO Box 204, York YO91 1XY, UK

\begin{abstract}
The aim of this research is to model the deformation and fracture behaviour of brittle wafers often used in chocolate confectionary products. Three point bending and compression experiments were performed on beam and circular disc samples respectively to determine the 'apparent' stress-strain curves in bending and compression. The deformation of the wafer for both these testing types was observed in-situ within an SEM. The wafer is modeled analytically and numerically as a composite material with a core which is more porous than the skins. X-ray tomography was used to generate a three dimensional volume of the wafer microstructure which was then meshed and used for quantitative analysis. A linear elastic material model, with a damage function and element deletion, was used and the XMT generated architecture was loaded in compression. The output from the FE simulations correlates closely to the load-deflection deformation observed experimentally.
\end{abstract}

Keywords: Wafer, foam, x-ray tomography, mechanical characterization, brittle fracture, finite element, damage PACS: Replace this text with PACS numbers; choose from this list: http://www.aip.org/pacs/index.html

\section{INTRODUCTION}

Wafers are often used in chocolate confectionary products. In large scale food production, of confectionary wafers with cream layered fillings, the cutting process is automated and one of the most crucial stages in the manufacturing. This often results in distorted cuts and broken wafers, leading to a loss of product and hence reduced processing efficiency. There is a need to develop models for predicting deformation and fracture of the wafers in such products so that problems arising during large scale processing can be eliminated. In this work, a comprehensive analysis of the wafer is being performed, using a combination of experimental, analytical and numerical techniques.

The wafer is made by baking between two hot plates, a liquid batter which consists primarily of wheat flour and water, with trace amounts of other ingredients. During the baking process most of the moisture evaporates, resulting in a porous foamed cellular structure. The faces of the wafer sheet that were in contact with the hot plates are less porous than the microstructure at the centre of the wafer sheet. The two denser faces of the wafer sheet are designated as the 'wafer skin' while the less dense region is referred to as the 'wafer core'.

\section{MECHANICAL TESTING}

Mechanical testing was performed using an Instron 5543 at a constant speed with a $1 \mathrm{kN}$ load cell. The wafer sheets were tested under compression and bending at ambient conditions, from which 'apparent' stress-strain curves were derived. Circular discs of $40 \mathrm{~mm}$ diameter were stacked on top of each other to test the stack size, reeding orientation and rate dependency of the wafer. The wafer deformation was shown to be independent of speed over 3 orders of magnitude and thus a single wafer sheet could safely be compressed at low speeds. Figure 1a) shows the 'apparent' stress-strain response of the wafer loaded in uni-axial compression. The three distinct regions of deformation, i.e. the elastic, plateau and densification zones, are observed. The elastic modulus was determined from the initial linear section of the curve and was found to be $3.6 \mathrm{MPa}$.

For the three point bending, single wafer specimens of $120 \mathrm{~mm}$ length and $15 \mathrm{~mm}$ width were used. Figure $1 \mathrm{~b}$ ) shows the brittle load-deflection response obtained from the three point bending experiments. The apparent flexural modulus was calculated using beam theory and was found to be $980 \mathrm{MPa}$. This value was considerably more than 
the compression modulus since the main deformation in bending occurred at the stiffer skins while in compression the porous core was the site of initial damage.
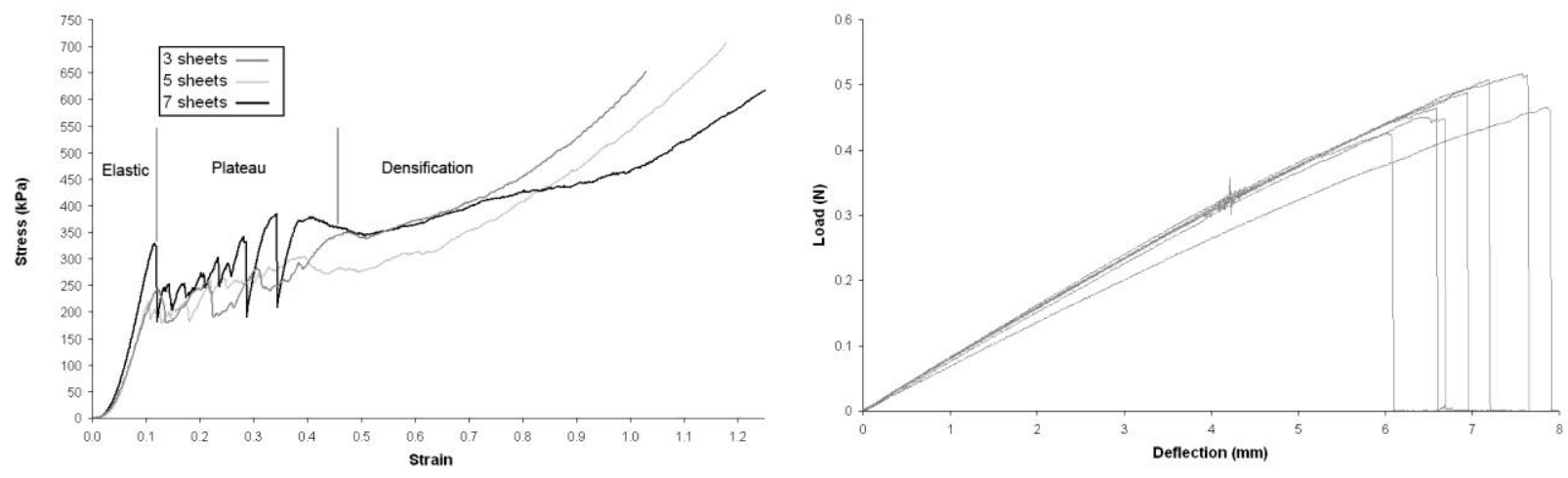

FIGURE 1. The stress-strain and load-displacement curves for a) compression and b) bending.

\section{MICROSTRUCTURE}

The wafer sheet was examined with an optical microscope and Scanning Electron Microscope to determine the wafer dimensions as well as to observe the internal microstructure. These cross-sectional images of the wafer sheet showed that the skins and the core had distinctively different microstructures. Pores were clearly visible in the skin but were considerably smaller compared to those in the core, suggesting that the core could be analysed as a closedcell foam while the skin could be considered as a porous solid with voids [1].

In-situ compression experiments were performed on the SEM so that the actual deformation could be observed within the microstructure of the wafer. As predicted, the initial fracture occurred within the cell walls of the core while the skins remained undamaged until the core had fully collapsed. As the wafer was compressed, a synchronized load-displacement graph was plotted and thus the distinct regions of the foam compression graph could be seen in terms of the material structure. In the linear elastic region it was difficult to see any cell wall buckling, but beyond the apparent yield point cracks were visibly propagating along the cell walls.
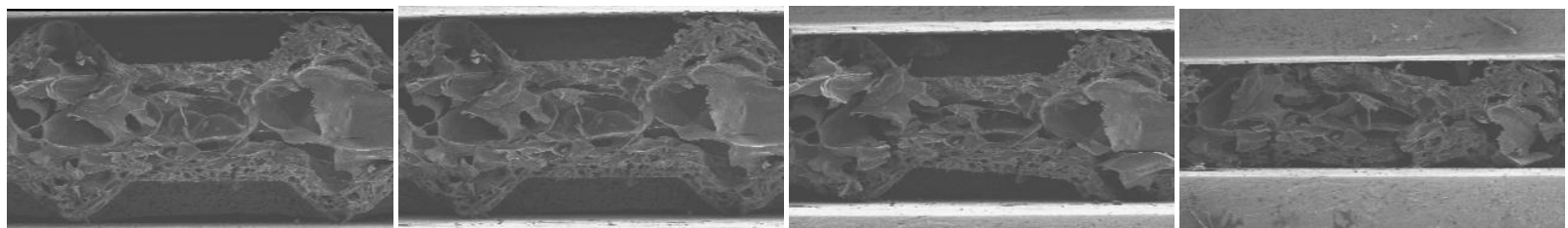

FIGURE 2. The microstructure at stages of compression a) initial, b) $\varepsilon=0.1$ (elastic), c) $\varepsilon=0.3$ (plateau), d) $\varepsilon=0.6$ (densification)

A Phoenix X-ray v|tome|x computerized tomography system was used to scan the wafer and produce a stack of image slices. They were then used to generate a 3D volume of the wafer microstructure using Avizo software [2] as shown in Fig 4. With this virtual wafer, it was possible to accurately determine the porous volume fraction of the overall wafer. This value was found to be $71 \%$, which was verified experimentally using helium pcynometry. The cell wall thickness was less than $5 \mu \mathrm{m}$ in some parts of the core while pore sizes varied between $300 \mu \mathrm{m}$ and 700 $\mu \mathrm{m}$.
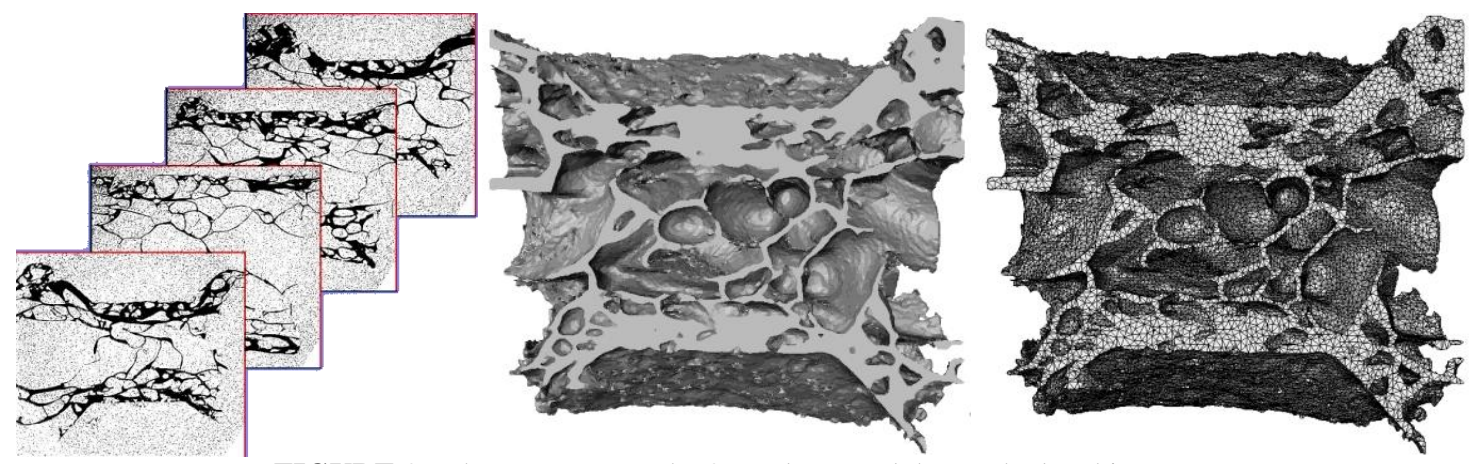

FIGURE 3. The XMT scans, the 3D volume and the meshed architecture. 
The reconstructed 3D volume was meshed with tetrahedral elements and then exported to the finite element software, Abaqus [3] to perform a quantitative analysis.

\section{ANALYTICAL WORK}

The wafer was analysed as a sandwich structure after microscopy showed that there were two distinct regions, namely the skin and the core. In compression, the wafer was treated as a composite, using the rule of mixtures. In bending, the wafer was initially considered as a sandwich beam with variable cross-section and then simplified to an I-beam of a single modulus [4]. Equations (1) for compression and (2) for bending, were solved simultaneously to calculate the modulus values of the skin $\left(E_{\text {skin }}\right)$ and core $\left(E_{\text {core }}\right)$ which were found to be $1710 \mathrm{MPa}$ and $1.73 \mathrm{MPa}$ respectively. $\mathrm{V}_{\mathrm{f}}$ represents the volume fraction of the core and skin and $\delta_{\text {central }}$ is the central deflection of the wafer beam.

$$
\begin{gathered}
E_{\text {compression }}=\frac{1}{\frac{V_{f}}{E_{\text {skin }}}+\frac{1-V_{f}}{E_{\text {core }}}} \\
\delta_{\text {central }}=\frac{1}{E_{\text {skin }}} \int_{0}^{L / 2} \frac{\frac{P x^{2}}{2}}{\left\{2\left[\frac{b h^{3}}{12}+b h\left(\frac{c}{2}+\frac{h}{2}\right)^{2}\right]+\left[\frac{b c^{3}}{12}\left(\frac{E_{\text {core }}}{E_{\text {skin }}}\right)\right]\right\}} \cdot d x
\end{gathered}
$$

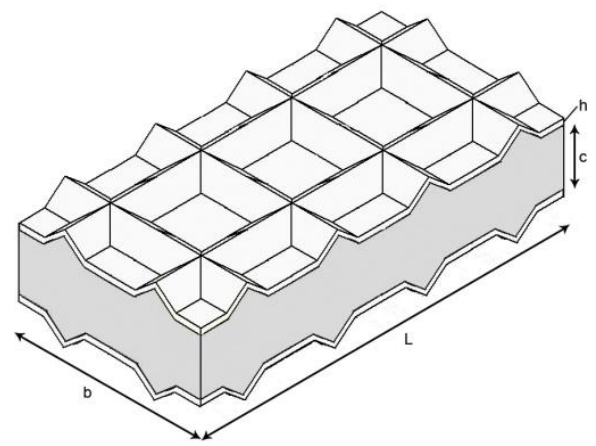

\section{NUMERICAL MODELLING}

The numerical model was developed to verify the analytical models using the finite element software Abaqus [3]. A three dimensional model of the wafer with a simple geometry was generated in CAD software and imported into Abaqus for analysis. The geometry, shown in Figure 4a), was sectioned like a sandwich to represent the wafer skins and core. The skin was assumed to be linear elastic while the core was described by the Crushable Foam material model [5]. The moduli used were the values calculated analytically while the input for Crushable Foam was taken from experimental data. The predicted 'apparent' stress-strain curve in compression is shown in Figure 4b) and compared to the experimental curve.
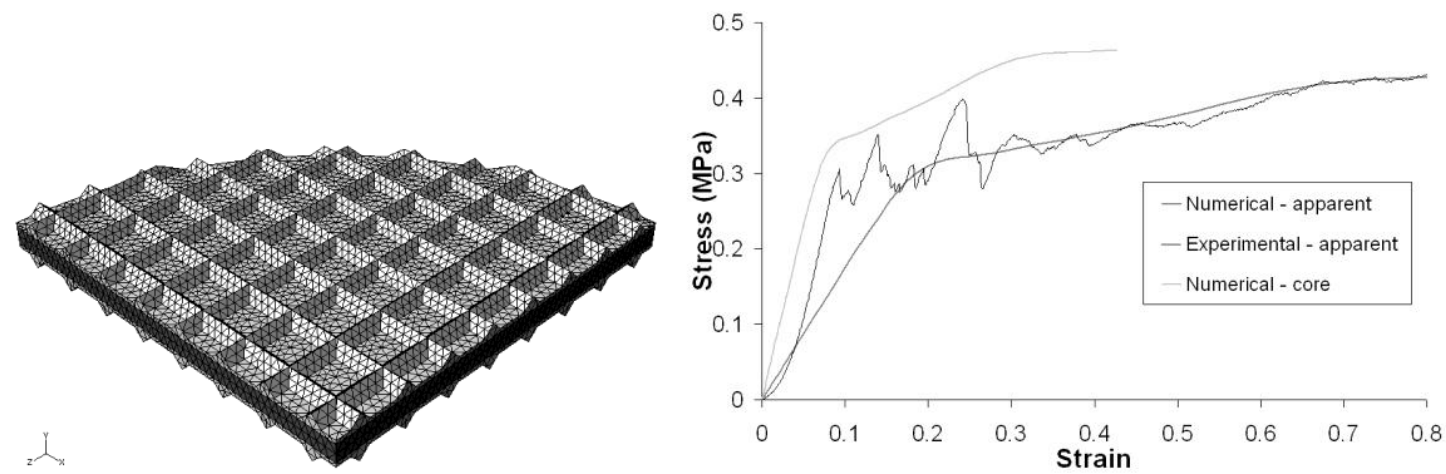

FIGURE 4. The a) simple geometry model with crushable foam core and b) the stress-strain graph.

The meshed 3D volume obtained from XMT was imported into Abaqus to simulate the compression of the exact architecture of the wafer. An elastic-plastic material model was used to represent the solid wafer. A damage criterion was implemented to the material model which degenerates and then deletes elements. The parameters for damage initiation and damage evolution were critical plastic strain and fracture energy respectively. The model was compressed between two rigid bodies which represented the compression plates.

The damage model selected from within the Abaqus material database was the 'Ductile Damage for Metals' material model [5]. It was implemented and then manipulated to simulate brittle fracture as observed in the wafer. It works in conjunction with the 'Elastic' and 'Plastic' material models and thus requires a Young's modulus, 
Poisson's ratio and yield stress. A graphical description of the entire material model can be seen in Figure 5a). The initial modulus $\left(\mathrm{E}_{\mathrm{s}}\right)$ and yield stress $\left(\sigma_{\mathrm{y} 0}\right)$ values were estimated using analytical equations for cellular solids [1]. These values were $200 \mathrm{MPa}$ and $25 \mathrm{MPa}$ respectively, with a Poisson's ratio of 0.3 . The fracture strain $\left(\varepsilon_{\mathrm{pl}}^{0}\right)$ was given a very small value $(0.001)$ so that the plastic deformation was negligible. The fracture energy $\left(\mathrm{G}_{\mathrm{f}}\right)$ was given a value of zero so that the element deletion was instantaneous once damage was initiated.
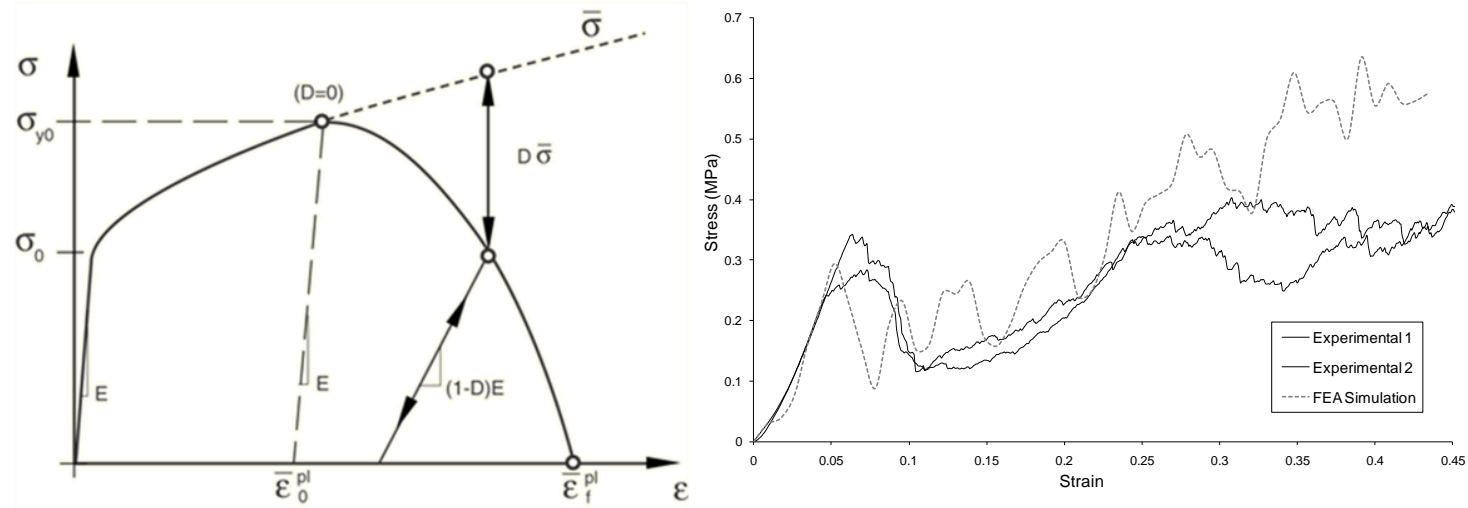

FIGURE 5. The a) stress-strain relationship of the material model and b) the deformation curve of the actual wafer architecture.

The deleted elements represented the brittle fracture of the cell walls of the wafer. As the model is compressed beyond initial fracture, the cell walls interact with each other due to self-contact of adjacent elements thus simulating the plateau region of the wafer deformation. The output deformation curve was compared to the experimental data as shown in Figure 5b). Figure 6 shows the wafer simulation at different stages during compression.
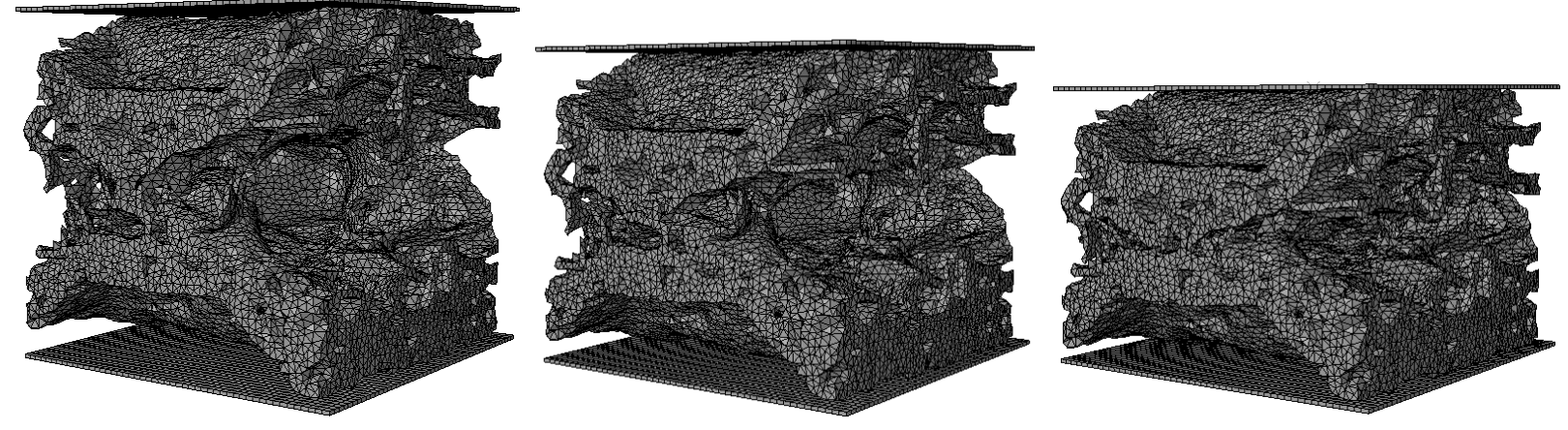

FIGURE 6. The FE model at stages of compression a) initial, b) $\varepsilon=0.1$ (elastic), c) $\varepsilon=0.3$ (plateau).

\section{CONCLUSION}

The wafer was tested mechanically in compression and bending loading configurations. These tests showed that the wafer behavior was characteristic of a brittle foam with an apparent modulus of $3.6 \mathrm{MPa}$ and $980 \mathrm{MPa}$ in compression and flexure respectively. The microstructure of the wafer was investigated using optical microscopy, scanning electron microscopy and x-ray tomography. It was observed that the structure of the wafer could be divided into 2 regions, with the core being a porous closed celled foam while the skins were considerably denser and stiffer. The overall porosity of the wafer was $71 \%$, determined experimentally and by 3D visual analysis. A finite element model of the actual wafer architecture was generated to simulate the experimental material behavior of the wafer. It utilized a damage criterion to simulate the brittle fracture of the cell walls. The FE output compared well to the experimental data, thus verifying that the model could be used to simulate cutting in the future.

\section{REFERENCES}

1. LJ Gibson, MF Ashby, Cellular Solids Structure and properties. 1st ed. Oxford: Pergamon Press, 1988.

2. http://www.tgs.com/products/avizo.asp

3. http://www.simulia.com/products/abaqus_fea.html

4. S Timoshenko, Strength of Materials Part 2 Advanced. 3rd ed. Canada: VNR, 1958.

5. ABAQUS/CAE User's Manual, Version 6.9-1. 